\title{
SELF-DUAL AND LOGARITHMIC REPRESENTATIONS OF THE TWISTED HEISENBERG-VIRASORO ALGEBRA AT LEVEL ZERO
}

\author{
DRAŽEN ADAMOVIĆ AND GORDAN RADOBOLJA
}

\begin{abstract}
This paper is a continuation of [2]. We present certain new applications and generalizations of the free field realization of the twisted Heisenberg-Virasoro algebra $\mathcal{H}$ at level zero. We find explicit formulas for singular vectors in certain Verma modules. A free field realization of self-dual modules for $\mathcal{H}$ is presented by combining a bosonic construction of Whittaker modules from [7] with a construction of logarithmic modules for vertex algebras. As an application, we prove that there exists a non-split self-extension of irreducible selfdual module which is a logarithmic module of rank two. We construct a large family of logarithmic modules containing different types of highest weight modules as subquotients. We believe that these logarithmic modules are related with projective covers of irreducible modules in a suitable category of $\mathcal{H}$-modules.
\end{abstract}

\section{INTRODUCTION}

The twisted Heisenberg-Virasoro Lie algebra $\mathcal{H}$ is an important example of a Lie algebra whose associated vertex algebra has many applications in the representation theory and conformal field theory. If the level of the corresponding Heisenberg vertex subalgebra is non-zero, the Heisenberg-Virasoro vertex algebra is isomorphic to the tensor product of Heisenberg vertex algebra and the (universal or simple) Virasoro vertex algebra (cf. [1], [14]). The study of the twisted Heisenberg-Virasoro algebra at level zero was initiated by Y. Billig [12] motivated by applications to the toroidal Lie algebras. New results on the representation theory were obtained in recent papers [2], [19], 24].

In this paper we continue our study of free field realization of the twisted HeisenbergVirasoro algebra from [2]. Our main motivation is to present free field realization of self-dual modules and certain Verma modules which we were unable to construct using methods from [2].

Let $\mathcal{H}$ be the twisted Heisenberg-Virasoro algebra at level zero. Let $V^{\mathcal{H}}\left(h, h_{I}\right)$ (resp. $L^{\mathcal{H}}\left(h, h_{I}\right)$ ) denote the Verma module (resp. the irreducible highest weight module) with highest weight $\left(c_{L}, c_{I}, c_{L, I}, h, h_{I}\right)$ such that $c_{I}=0, c_{L, I} \neq 0$ is fixed but arbitrary, and with highest weight vector $v_{h, h_{I}}$. Then $V^{\mathcal{H}}\left(h, h_{I}\right)$ is reducible if and only if $h_{I} / c_{L, I}-1 \in \mathbb{Z} \backslash\{0\}$ (cf. [12]).

A free field realization of irreducible highest weight modules for the twisted HeisenbergVirasoro algebra at level zero was presented by the authors in [2]. Using free field realization we calculated the fusion rules for non-generic irreducible modules i.e., for those irreducible modules which are not isomorphic to a Verma $\mathcal{H}$-module. In our approach, the screening

2000 Mathematics Subject Classification. Primary 17B69, Secondary 17B67, 17B68, 81R10.

Key words and phrases. Heisenberg-Virasoro algebra, logarithmic representations, Whittaker modules, self-dual modules, singular vectors. 
operator $Q$ introduced in [2, Section 2] has played an important role. In particular, the singular vector in $V^{\mathcal{H}}\left(h, h_{I}\right)$ in the case

$$
h_{I} / c_{L, I}-1=p \in \mathbb{Z}_{>0}
$$

is expressed as $Q v_{h+p, h_{I}}=S_{p}(c) v_{h, h_{I}}$ where $S_{p}(c)$ is $p^{t h}$-Schur polynomial in variables $(c(-1), c(-2), \cdots)$ defined by (4.16) where $c=-I / c_{L, I}$ :

$$
S_{p}(c)=S_{p}(c(-1), \ldots, c(-p))=S_{p}\left(\frac{-1}{c_{L, I}} I(-1), \ldots, \frac{-1}{c_{L, I}} I(-p)\right)
$$

(see (2.7) and [2, Theorem 4.3]).

In the present paper we found a similar approach for the singular vector in the case

$$
h_{I} / c_{L, I}-1=-p \quad\left(p \in \mathbb{Z}_{>0}\right) .
$$

The formula is

$$
\begin{aligned}
& \sum_{i=1}^{p}\left(L(-i) S_{p-i}(-c)\right) v_{h, h_{I}}+\left(h+\frac{c_{L}-2}{24}(p-1)\right) S_{p}(-c) v_{h, h_{I}} \\
& -\frac{c_{L}-26}{24}\left(\sum_{i=1}^{p}(i-1) c(-i) S_{p-i}(-c)\right) v_{h, h_{I}} .
\end{aligned}
$$

Verma modules $V^{\mathcal{H}}\left(h, h_{I}\right)$ in the case (1.1) are explicitly constructed in Section 5 by using certain deformation studied previously in 44. Singular vector (1.2) admits a nice interpretation as an element $e^{\frac{p-1}{2} d^{2}+\frac{1-r}{2} c+c}$ of the group algebra in the explicit lattice realization (cf. Theorem 5.3.

It turned out that the methods from [2] do not provide a free field realization of self-dual modules $L^{\mathcal{H}}\left(h, h_{I}\right)$ such that $h \neq \frac{c_{L}-2}{24}, h_{I}=c_{L, I}$. On the other hand, the same paper contains certain results on vanishing of the fusion rules in the category of modules which contains self-dual module $L^{\mathcal{H}}\left(h, h_{I}\right)$ as above (see [2, Theorem 5.4(3), Remark 6.5]). In order to understand these fusion rules properties from the vertex-algebraic point of view, one needs to find a bosonic realization of self-dual modules. We shall see that the realization includes both the concepts of Whittaker and logarithmic modules for vertex algebras. We shall prove that the Whittaker module $\Pi_{\lambda}$ for the vertex algebra $\Pi(0)$ introduced in [7, after certain logarithmic deformation (cf. Theorem $\left[5.2\right.$ ) becomes a highly reducible $\mathcal{H}$-module $\widetilde{\Pi}_{\lambda}$ which contains $L^{\mathcal{H}}\left(h, h_{I}\right)$ as a submodule. Since $\widetilde{\Pi}_{\lambda}$ is not a module for the Heisenberg vertex algebra $M(1)$, it is clear that such module could not have appeared in the fusion rules analysis made in [2].

In Section 2 we recall from [2] a free field realization of $\mathcal{H}$, the definition of vertex algebra $\Pi(0)$ and its modules $\Pi(p, r)$. We study an extension of the Heisenberg-Virasoro vertex algebra $\overline{\Pi(0)} \subset \Pi(0)$ and present a structure of $\overline{\Pi(0)}$-modules $\Pi(p, r)$ in Section 3 , By using certain relation in $\Pi(0)$-modules we recover formula 1.2 in Section 4 . Then we consider a deformed action of $\mathcal{H}$ on these modules and obtain a family of modules $\widetilde{\Pi(p, r)}[\mathrm{cf}$. Theorem 5.2. Theorem 5.5 with the following properties:

- $\widetilde{\Pi(p, r)}$ is a logarithmic $\mathcal{H}$-module with the following action of the element $\widetilde{L(0)}$ of the Virasoro algebra: 
$-\mathbb{C}[\widetilde{L(0)}] v$ is finite-dimensional for every $v \in \widetilde{\Pi(p, r)}, p \geq 1$,

- $\mathbb{C}[\widetilde{L(0)}] v$ is infinite-dimensional for every $v \in \widetilde{\Pi(p, r)}, p \leq 0$.

- For $p \geq 1, \widetilde{\Pi(p, r)}$ admits a filtration

$$
\widetilde{\Pi(p, r)} \cong \bigcup_{m \geq 0} Z^{(m)},
$$

such that $Z^{(m)}$ is a logarithmic $\mathcal{H}$-module of rank $m+1$ and $Z^{(m)} / Z^{(m-1)}$ is a weight $\mathcal{H}$-module. [cf. Theorem 5.4]

In Section [6] we consider a deformed action on Whittaker $\Pi(0)$-modules and obtain a realization of self-dual $\mathcal{H}$-modules $L^{\mathcal{H}}\left(h, c_{L, I}\right), h \neq \frac{c_{L}-2}{24}$ [cf. Theorem 6.3]

Finally, we find new applications of our results on the vertex algebra associated to the $W(2,2)$-algebra. We present a non-local bosonic formula (7.26) for the screening operator introduced by the authors in [3. We hope that our new expression for screening operator can be applied to construction of a quantum group which would play the role of Kazhdan-Lusztig dual of the vertex algebra $W(2,2)$.

We also discuss a connection of our approach with a realization of the $\mathrm{BMS}_{3}$-algebra obtained in [11] in the case $c_{L}=26$.

Acknowledgements. The authors are partially supported by the Croatian Science Foundation under the project 2634 and by the QuantiXLie Centre of Excellence, a project cofinanced by the Croatian Government and European Union through the European Regional Development Fund - the Competitiveness and Cohesion Operational Programme (Grant KK.01.1.1.01.0004). We thank the referee for his/her valuable comments.

\section{Results from [2]}

In this section we recall from [2] the free field realization of the twisted Heisenberg-Virasoro algebra, the construction of vertex algebra $\Pi(0)$ and their modules $\Pi(p, r)$. The definition of the lattice is slightly changed, but the action of the generators of the Heisenberg-Virasoro algebra is the same as in [2]. Main results of Section 2 of [2] are stated in Proposition 2.1.

We also present new, explicit formulas for sub-singular vectors introduced in [2, Proposition 2.7].

Recall that the twisted Heisenberg-Virasoro algebra is an infinite-dimensional complex Lie algebra $\mathcal{H}$ with basis

$$
\{L(n), I(n): n \in \mathbb{Z}\} \cup\left\{C_{L}, C_{L I}, C_{I}\right\}
$$

and commutation relation:

$$
\begin{aligned}
& {[L(n), L(m)]=(n-m) L(n+m)+\delta_{n,-m} \frac{n^{3}-n}{12} C_{L},} \\
& {[L(n), I(m)]=-m I(n+m)-\delta_{n,-m}\left(n^{2}+n\right) C_{L I},} \\
& {[I(n), I(m)]=n \delta_{n,-m} C_{I},} \\
& {\left[\mathcal{H}, C_{L}\right]=\left[\mathcal{H}, C_{L I}\right]=\left[\mathcal{H}, C_{I}\right]=0 .}
\end{aligned}
$$

Let $V^{\mathcal{H}}\left(c_{L}, c_{I}, c_{L, I}, h, h_{I}\right)$ denote the Verma module with highest weight $\left(c_{L}, c_{I}, c_{L, I}, h, h_{I}\right)$, and $L^{\mathcal{H}}\left(c_{L}, c_{I}, c_{L, I}, h, h_{I}\right)$ its irreducible quotient (cf. [12]). In this paper we consider the case 
$c_{I}=0$. For simplicity we shall denote the Verma module $V^{\mathcal{H}}\left(c_{L}, 0, c_{L, I}, h, h_{I}\right)$ with $V^{\mathcal{H}}\left(h, h_{I}\right)$ and its irreducible quotient with $L^{\mathcal{H}}\left(h, h_{I}\right)$.

Define the following hyperbolic lattice $H y p=\mathbb{Z} x+\mathbb{Z} y$ such that

$$
\langle x, x\rangle=\langle y, y\rangle=0,\langle x, y\rangle=1 .
$$

Let $\mathfrak{h}=\mathbb{C} \otimes_{\mathbb{Z}} H y p$ and extend the form $\langle\cdot, \cdot\rangle$ on $\mathfrak{h}$. We can consider $\mathfrak{h}$ as an abelian Lie algebra. Let $\widehat{\mathfrak{h}}=\mathbb{C}\left[t, t^{-1}\right] \otimes \mathfrak{h} \oplus \mathbb{C} K$ be the affinization of $\mathfrak{h}$. Let $\gamma \in \mathfrak{h}$ and consider $\widehat{\mathfrak{h}}$-module

$$
M(1, \gamma):=U(\widehat{\mathfrak{h}}) \otimes_{U(\mathbb{C}[t] \otimes \mathfrak{h} \oplus \mathbb{C} K)} \mathbb{C}
$$

where $t \mathbb{C}[t] \otimes \mathfrak{h}$ acts trivially on $\mathbb{C}, \mathfrak{h}$ acts as $\langle\delta, \gamma\rangle$ for $\delta \in \mathfrak{h}$ and $K$ acts as 1 . We shall denote the highest weight vector in $M(1, \gamma)$ by $e^{\gamma}$.

We shall write $M(1)$ for $M(1,0)$. For $h \in \mathfrak{h}$ and $n \in \mathbb{Z}$ we write $h(n)$ for $t^{n} \otimes h$. Set $h(z)=\sum_{n \in \mathbb{Z}} h(n) z^{-n-1}$. Then $M(1)$ is a vertex algebra which is generated by the fields $h(z), h \in \mathfrak{h}$ (cf. [20]. Moreover, $M(1, \gamma)$ for $\gamma \in \mathfrak{h}$, are irreducible $M(1)$-modules.

Let $V_{H y p}=M(1) \otimes \mathbb{C}[H y p]$ be the vertex algebra associated to the lattice Hyp, where $\mathbb{C}[$ Hyp $]$ is the group algebra of Hyp.

In this paper we shall consider the lattice $L=\mathbb{Z} c+\mathbb{Z} d$ such that $c=x$ and $d=2 y$. Then $V_{L}$ is a vertex subalgebra of $V_{\text {Hyp }}$.

Define the Heisenberg and the Virasoro vector:

$$
\begin{aligned}
I & =-c_{L, I} c(-1), \\
\omega & =\frac{1}{2} c(-1) d(-1)+\frac{c_{L}-2}{24} c(-2)-\frac{1}{2} d(-2) .
\end{aligned}
$$

Then the components of the fields

$$
I(z)=Y(I, z)=\sum_{n \in \mathbb{Z}} I(n) z^{-n-1}, \quad L(z)=Y(\omega, z)=\sum_{n \in \mathbb{Z}} L(n) z^{-n-2}
$$

satisfy the commutation relations for the twisted Heisenberg-Virasoro Lie algebra $\mathcal{H}$ and $I$ and $\omega$ generate the simple Heisenberg-Virasoro vertex algebra $L^{\mathcal{H}}\left(c_{L}, 0, c_{L, I}, 0,0\right)$ which we shall denote by $L^{\mathcal{H}}\left(c_{L}, c_{L, I}\right)$.

We have:

$$
\begin{aligned}
& {[L(n), c(m)]=-m c(n+m)+\left(m^{2}-m\right) \delta_{m+n, 0}} \\
& {[L(n), d(m)]=-m d(n+m)-\frac{c_{L}-2}{12}\left(m^{2}-m\right) \delta_{m+n, 0}}
\end{aligned}
$$

Let $u=e^{c}$. Then by [2, Lemma 2.4]

$$
Q=\operatorname{Res}_{z} Y(u, z)=u_{0} .
$$

is a screening operator. This means that

$$
[Q, I(n)]=[Q, L(n)]=0 \quad \forall n \in \mathbb{Z},
$$

i.e., $Q$ commutes with the action of the Heisenberg-Virasoro algebra.

Recall that screening operators provide an important tool for construction of singular vectors in free-field realizations (cf. [26]).

One can show that

$$
\Pi(0)=M(1) \otimes \mathbb{C}[\mathbb{Z} c]
$$


is a simple vertex algebra (cf. [13]). Let $Y(\cdot, z)$ be the associated vertex operator. The vertex operator $Y(u, z)$ and the screening operator $Q$ are well-defined on every $\Pi(0)$-module.

Let

$$
d^{1}=d+\frac{c_{L}-26}{12} c, \quad d^{2}=d-\frac{c_{L}-26}{12} c .
$$

Consider the following irreducible $\Pi(0)$-modules

$$
\Pi(p, r):=\Pi(0) \cdot e^{\frac{p-1}{2} d^{2}+\frac{1-r}{2} c} \quad \text { where } \quad p \in \mathbb{Z}, r \in \mathbb{C} .
$$

(The irreducibility of $\Pi(p, r)$ was also proved in [13]).

Let

$$
\begin{aligned}
& h_{p, r}=\left(1-p^{2}\right) \frac{c_{L}-26}{24}+1-p+(1-r) \frac{p}{2} . \\
& W_{p, r}=U(\mathcal{H}) \cdot e^{\frac{p-1}{2} d^{2}+\frac{1-r}{2} c} \subset \Pi(p, r) .
\end{aligned}
$$

Set $v_{p, r}=e^{\frac{p-1}{2} d^{2}+\frac{1-r}{2} c}$. Note that $h_{p, r+2}=h_{p, r}-p$.

The following result is proved in Propositions 2.5 and 2.7 of [2].

Proposition 2.1. We have:

(1) $W_{p, r} \cong V^{\mathcal{H}}\left(h_{p, r},(1-p) c_{L, I}\right)$ if and only if $p \in \mathbb{C} \backslash \mathbb{Z}_{\geq 1}$.

(2) $W_{p, r} \cong L^{\mathcal{H}}\left(h_{p, r},(1-p) c_{L, I}\right)$ if $p \in \mathbb{Z}_{\geq 1}$.

(3) For every $r \in \mathbb{C}, W_{0, r} \cong L^{\mathcal{H}}\left(\frac{c_{L}-2}{24}, c_{L, I}\right)$.

The following modules were not constructed in [2]:

- Reducible Verma modules $V^{\mathcal{H}}\left(h_{p, r},(1-p) c_{L, I}\right)$ in the case $p \in \mathbb{Z}_{\geq 1}$.

- Self-dual modules $L^{\mathcal{H}}\left(h, c_{L, I}\right)$ for $h \neq \frac{c_{L}-2}{24}$.

We shall present the construction of these modules in Sections 5 and 6

We shall also need the following result.

Lemma 2.2. Assume that $p \in \mathbb{Z}_{>0}$. As an $\mathcal{H}$-module, $\Pi(p, r)$ is generated by a family of $\mathcal{H}$-singular vectors $\left\{v_{p, r-2 \ell} \mid \ell \in \mathbb{Z}\right\}$ and a family of $\mathcal{H}$-cosingular vectors $\left\{v_{p, r-2 \ell}^{(m)} \mid \ell, m \in\right.$ $\mathbb{Z}, m \geq 1\}$, where

$$
v_{p, r-2 \ell}^{(m)}=\frac{(-1)^{m}}{2^{m} m !} d(-p)^{m} v_{p, r-2 \ell}
$$

Proof. By [2, Proposition 2.7] we have that $\Pi(p, r)$ is generated as $\mathcal{H}$-module by a family of singular vectors $\left\{v_{p, r-2 \ell} \mid \ell \in \mathbb{Z}\right\}$ and by a family of cosingular vectors $\left\{w_{p, r-2 \ell}^{(m)} \mid \ell, m \in\right.$ $\mathbb{Z}, m \geq 1\}$ satisfying

$$
Q^{m} w_{p, r-2 \ell}^{(m)}=v_{p, r-2(\ell+m)} .
$$

Let us prove that for cosingular vectors $w_{p, r-2 \ell}^{(m)}$ we may choose $v_{p, r-2 \ell}^{(m)}$.

Recall the commutator relations in $\Pi(0)$ :

$$
\left[d(-k), e_{j}^{c}\right]=2 e_{j-k}^{c}, \quad\left[e_{j}^{c}, c(-k)\right]=0, \quad\left[e_{0}^{c}, e_{j}^{c}\right]=0 .
$$

By induction on $m$ we see that $e_{-k p}^{c} v_{p, r-2 \ell}^{(m)}$ is spanned by $S_{(k+i-1) p}(c) v_{p, r-2(\ell+1)}^{(m-i)}, i=k-$ $1, \ldots m$ for each $k \in \mathbb{Z}_{\geq 0}$. Therefore we have

$$
Q^{j} v_{p, r-2 \ell}^{(m)}= \begin{cases}v_{p, r-2(\ell+j)}^{(m-j)} \bmod \operatorname{Ker}_{\Pi(p, r)} Q^{m}, & j<m, \\ v_{p, r-2(\ell+m)}, & j=m .\end{cases}
$$


The proof follows.

See Figure 1 in the Appendix $\mathrm{A}$ for structure of $\Pi(0)$-module $\Pi(p, r)$.

Remark 2.3. Note that $\Pi(p, r)=\Pi(p, s)$ if and only if $r-s \in 2 \mathbb{Z}$. Moreover, $\Pi(1,1)=\Pi(0)$ and $U(\mathcal{H}) v_{1,1} \cong L^{\mathcal{H}}\left(c_{L}, c_{L, I}\right)$.

\section{An extension of the vertex Algebra $L^{\mathcal{H}}\left(c_{L}, c_{L, I}\right)$}

An extension of the Heisenberg-Virasoro vertex algebra Now we study modules $\Pi(p, r)$ in more details. There is a vertex subalgebra of $\Pi(0)$ which can be treated as an extension of the vertex algebra $L^{\mathcal{H}}\left(c_{L}, c_{L, I}\right)$ :

$$
\overline{\Pi(0)}=\operatorname{Ker}_{\Pi(0)} Q .
$$

In this section we obtain filtrations of $\Pi(p, r)$ by $\overline{\Pi(0)}$-modules such that the subquotients are irreducible over $\overline{\Pi(0)}$.

Proposition 3.1. Let $\Pi(0)^{(n)}=\operatorname{Ker}_{\Pi(0)} Q^{n+1}$. Then we have

(1) $\overline{\Pi(0)}$ is a vertex subalgebra of $\Pi(0)$ which is as $L^{\mathcal{H}}\left(c_{L}, c_{L, I}\right)$-module isomorphic to

$$
\overline{\Pi(0)}=\bigoplus_{n \in \mathbb{Z}} W_{1,1-2 n} \cong \bigoplus_{n \in \mathbb{Z}} L^{\mathcal{H}}(n, 0)
$$

(2) For every $n \in \mathbb{Z}_{\geq 0}, \Pi(0)^{(n)}$ and $\Pi(0)^{(n+1)} / \Pi(0)^{(n)}$ are $\overline{\Pi(0)}$-modules. Moreover we have

$$
\Pi(0)=\bigcup_{n \geq 0} \Pi(0)^{(n)}, \quad \Pi(0)^{(n)} \cdot \Pi(0)^{(m)} \subset \Pi(0)^{(n+m)} .
$$

Proof. Since $Q$ is a screening operator, $\overline{\Pi(0)}=\operatorname{Ker}_{\Pi(0)} Q$ is a vertex subalgebra of $\Pi(0)$. By using [2, Proposition 2.7] we get

$$
\begin{aligned}
\overline{\Pi(0)} & =\bigoplus_{n \in \mathbb{Z}} L^{\mathcal{H}}\left(c_{L}, c_{L, I}\right) \cdot e^{n c} \\
& =\bigoplus_{n \in \mathbb{Z}} W_{1,1-2 n} .
\end{aligned}
$$

The proof of assertion (2) is clear.

Condition (2) from Proposition 3.1 shows that $\mathcal{H}$-modules (and $\overline{\Pi(0)}$-modules) $\Pi(0)^{(n)}$ give a $\mathbb{Z}_{\geq 0}$ filtration on the vertex algebra $\Pi(0)$. In the same way we can construct a filtration on certain $\Pi(0)$-modules.

Theorem 3.2. Assume that $p \in \mathbb{Z}_{>0}$. Let $\Pi(p, r)^{(m)}=\operatorname{Ker}_{\Pi(p, r)} Q^{m+1}$. Then we have

(1) $\Pi(p, r) \cong \bigcup_{m \geq 0} \Pi(p, r)^{(m)}, \quad \Pi(0)^{(n)} \cdot \Pi(p, r)^{(m)} \subset \Pi(p, r)^{(n+m)}$.

(2) For every $m \in \mathbb{Z}_{\geq 0} \Pi(p, r)^{(m)}$ is $\overline{\Pi(0)}$-module and $\Pi(p, r)^{(m)} \subset \Pi(p, r)^{(m+1)}$.

(3) $\Pi(p, r)^{(m+1)} / \Pi(p, r)^{(m)}$ is an irreducible $\overline{\Pi(0)}$-module which is as $\mathcal{H}$-module isomorphic to

$$
\bigoplus_{n \in \mathbb{Z}} W_{p, r-2 n} \cong \bigoplus_{n \in \mathbb{Z}} L^{\mathcal{H}}\left(h_{p, r}+n p,(1-p) c_{L, I}\right)
$$


Proof. The proof of assertions (1) and (2) is clear. The decomposition (3.14) essentially follows from [2, Proposition 2.7]. Let us prove the irreducibility result in (3). It suffices to prove that

$$
W_{1,1-2 n} \cdot W_{p, r-2 \ell}=W_{p, 1-2(n+\ell)}
$$

Recall that

$$
W_{1,1-2 n}=U(\mathcal{H}) \cdot e^{n c}, \quad W_{p, r-2 \ell}=U(\mathcal{H}) \cdot u_{p, r-2 \ell} \bmod \Pi(p, r)^{(m)},
$$

where $u_{p, r-2 \ell}$ is an $\mathcal{H}$-cosingular vector $v_{p, r-2(\ell-m-1)}^{(m+1)}$ such that $Q^{m+1} u_{p, r-2 \ell}=v_{p, r-2 \ell}$.

Since $e_{k_{0}}^{n c} v_{p, r-2 \ell}=v_{p, r-2(\ell+n)}$ for $k_{0}=-n(p-1)-1$ we get that

$$
Q^{m+1} e_{k_{0}}^{n c} u_{p, r-2 \ell}=v_{p, r-2(\ell+n)} \neq 0 .
$$

So $e_{k_{0}}^{n c} u_{p, r-2 \ell}+\Pi(p, r)^{(m)}$ generates $W_{p, 1-2(n+\ell)}$ in $\Pi(p, r)^{(m+1)} / \Pi(p, r)^{(m)}$. The proof follows.

Figure 1 in Appendix $\AA$ represents a portion of module $\Pi(p, r)$ with action of $Q$ and $e_{-p}^{c}$ on (sub)singular generators obtained in Lemma 2.2. Quotient module $\Pi(p, r)^{(m+1)} / \Pi(p, r)^{(m)}$ is a direct sum of "slices", each generated by $v_{p, r-2 \ell}^{(m+1)}+\Pi(p, r)^{(m)}$ and isomorphic to $W_{p, r-2(\ell+m+1)}$.

Let us now consider modules $\Pi(-p, r)$. Recall that $h_{-p, r+2}=h_{-p, r}+p$.

\section{Theorem 3.3.}

(1) As an $\mathcal{H}$-module $\Pi(0, r)$ is isomorphic to

$$
\bigoplus_{n \in \mathbb{Z}} W_{0, r} \cong \bigoplus_{n \in \mathbb{Z}} L^{\mathcal{H}}\left(\frac{c_{L}-2}{24}, c_{L, I}\right) .
$$

(2) Let $p \in \mathbb{Z}_{>0}$. Consider $Q$ as $\overline{\Pi(0)}$-endomorphism of $\Pi(-p, r)$, and let $\Pi(-p, r)^{(m)}=$ $\operatorname{Im} Q^{m}$. Then for $m \in \mathbb{Z}_{>0}$ we have

(a) $\Pi(-p, r) \cong \Pi(-p, r)^{(m)}$ as $\overline{\Pi(0)}$-modules,

(b) $\Pi(-p, r)^{(m)} / \Pi(-p, r)^{(m+1)}$ is an irreducible $\overline{\Pi(0)}$-module which is as an $\mathcal{H}$-module isomorphic to

$$
\bigoplus_{\ell \in \mathbb{Z}} L^{\mathcal{H}}\left(h_{-p, r}+\ell p,(1+p) c_{L, I}\right)
$$

Proof. It was shown in [2] that $\Pi(p, r) \cong \bigoplus_{\ell \in \mathbb{Z}} W_{p, r+2 \ell}$ as $\mathcal{H}$-module when $p \notin \mathbb{Z}_{>0}$. Decomposition in (1) then follows from Proposition 2.1 (3). Since

$$
Q v_{-p, r}=S_{p}(c) v_{-p, r-2}
$$

we see that $Q\left(W_{-p, r+2 \ell}\right) \subset W_{-p, r+2(\ell-1)}$ and since $Q$ commutes with the action of $\mathcal{H}$ we have $\operatorname{Ker} Q=0$. Therefore $\Pi(-p, r) \cong \operatorname{Im} Q=\Pi(-p, r)^{(1)}$, so claim (a) follows by iteration.

Let us prove assertion (b). It suffices to prove the claim for $m=0$. General statement then follows from (a). Recall that $W_{-p, r+2 \ell} \cong V^{\mathcal{H}}\left(h_{-p, r+2 \ell},(1+p) c_{L, I}\right)$ and notice that $Q v_{-p, r+2(\ell+1)}=S_{p}(c) v_{-p, r+2 \ell}$ is an $\mathcal{H}$-singular vector in $W_{-p, r+2 \ell}$ which generates the maximal submodule. Now $U(\mathcal{H}) \cdot\left(v_{-p, r+2 \ell}+\operatorname{Im} Q\right) \cong L^{\mathcal{H}}\left(h_{-p, r+2 \ell},(1+p) c_{L, I}\right)$. This proves the decomposition in (b). Proof of irreducibility in (b) is similar to the proof of Theorem 3.2 .

See Figure 2 in Appendix $\mathrm{A}$ for reference. 


\section{Relations in $\Pi(0)$-Modules}

In this section we shall apply a relation in the vertex algebra $\Pi(0)$ on its modules and recover an explicit formula for a singular vector in the Verma module $V^{\mathcal{H}}\left(h,(1-p) c_{L, I}\right)$, for $p \geq 1$. We shall use this formula in Section 5 when we construct a free field realization of these Verma modules.

Recall first that the Schur polynomials $S_{r}\left(x_{1}, x_{2}, \ldots\right)$ in variables $x_{1}, x_{2}, \ldots$ are defined by

$$
\exp \left(\sum_{n=1}^{\infty} \frac{x_{n}}{n} y^{n}\right)=\sum_{r=1}^{\infty} S_{r}\left(x_{1}, x_{2}, \ldots\right) y^{r}
$$

Then for any $\gamma \in L$ we set $S_{r}(\gamma):=S_{r}(\gamma(-1), \gamma(-2), \ldots)$.

Let

$$
e^{-c}(z)=Y\left(e^{-c}, z\right)=\sum_{i \in \mathbb{Z}} e_{i}^{-c} z^{-i-1}
$$

By direct calculation we get

$$
L(-2) e^{-c}=\frac{c_{L}-26}{24} c(-2) e^{-c}-\frac{1}{2} L(-1)\left(d(-1) e^{-c}\right) .
$$

Let

$$
s=\left(L(-2)-\frac{c_{L}-26}{24} c(-2)\right) e^{-c} .
$$

Then we get

Lemma 4.1. On every $\Pi(0)$-module we have

$$
\begin{aligned}
\mathcal{Q}= & s_{0}=\operatorname{Res}_{z} Y(s, z) \\
= & \sum_{i=0}^{\infty}\left(L(-2-i) e_{i}^{-c}+e_{-i-1}^{-c} L(-1+i)\right) \\
& \quad-\frac{c_{L}-26}{24} \sum_{i \in \mathbb{Z}}(i+1) c(-i-2) e_{i}^{-c} \\
= & 0 .
\end{aligned}
$$

Proof. The assertion follows from (4.17) and the fact that

$$
(L(-1) u)_{0}=0
$$

in every vertex operator algebra.

Now we shall see some consequences of the relation $\mathcal{Q}=0$ for irreducible $\mathcal{H}$-modules $L^{\mathcal{H}}\left(h, h_{I}\right)$ such that $h_{I}=(1-p) c_{L, I}, p \in \mathbb{Z}_{>0}$, which are realized as $W_{p, r}$, for $r \in \mathbb{C}$. 
We have

$$
\begin{aligned}
0= & \mathcal{Q}^{\frac{p-1}{2} d^{2}+\frac{1-r}{2} c} \\
= & \left(\sum_{i=0}^{\infty}\left(L(-2-i) e_{i}^{-c}\right)+e_{-1}^{-c} L(-1)+h_{p, r} e_{-2}^{-c}\right) e^{\frac{p-1}{2} d^{2}+\frac{1-r}{2} c} \\
& -\frac{c_{L}-26}{24}\left(\sum_{i \in \mathbb{Z}}(i+1) c(-i-2) e_{i}^{-c}\right) e^{\frac{p-1}{2} d^{2}+\frac{1-r}{2} c} \\
= & \left(\sum_{i=0}^{p-2}\left(L(-2-i) e_{i}^{-c}\right)+e_{-1}^{-c} L(-1)+h_{p, r} e_{-2}^{-c}\right) e^{\frac{p-1}{2} d^{2}+\frac{1-r}{2} c} \\
& -\frac{c_{L}-26}{24}\left(\sum_{i=-2}^{p-2}(i+1) c(-i-2) e_{i}^{-c}\right) e^{\frac{p-1}{2} d^{2}+\frac{1-r}{2} c} \\
= & \left(\sum_{i=1}^{p} L(-i) e_{i-2}^{-c}+\left(h_{p, r}-1+\frac{c_{L}-26}{24}(p-1)\right) e_{-2}^{-c}\right. \\
& \left.-\frac{c_{L}-26}{24} \sum_{i=-1}^{p-2}(i+1) c(-i-2) e_{i}^{-c}\right) e^{\frac{p-1}{2} d^{2}+\frac{1-r}{2} c} \\
= & \left(\sum_{i=1}^{p} L(-i) S_{p-i}(-c)+\left(h_{p, r}-1+\frac{c_{L}-26}{24}(p-1)\right) S_{p}(-c)\right. \\
& \left.-\frac{c_{L}-26}{24} \sum_{i=1}^{p}(i-1) c(-i) S_{p-i}\right) e^{\frac{p-1}{2} d^{2}+\frac{1-(r+2)}{2} c}
\end{aligned}
$$

In this way we have proved:

Proposition 4.2. Let $p \in \mathbb{Z}_{>0}$. In $W_{p, r+2}$ we have:

$$
\begin{aligned}
0= & \left(\left(\sum_{i=1}^{p} L(-i) S_{p-i}(-c)\right)+\left(h_{p, r}-1+\frac{c_{L}-26}{24}(p-1)\right) S_{p}(-c)+\right. \\
& \left.-\frac{c_{L}-26}{24}\left(\sum_{i=1}^{p}(i-1) c(-i) S_{p-i}(-c)\right)\right) v_{p, r+2}
\end{aligned}
$$

In particular, let $h_{I}=(1-p) c_{L, I}, h=h_{p, r+2}$. Then the singular vector of level $p$ in $V^{\mathcal{H}}\left(h, h_{I}\right)$ is $\Phi_{p}(L, c) \cdot v_{h, h_{I}}$ where

$$
\begin{aligned}
& \Phi_{p}(L, c):=\sum_{i=1}^{p}\left(L(-i) S_{p-i}(-c)\right)+S_{p}(-c)\left(L(0)+\frac{c_{L}-2}{24}(p-1)\right) \\
& \left.-\frac{c_{L}-26}{24}\left(\sum_{i=1}^{p}(i-1) c(-i) S_{p-i}(-c)\right)\right)
\end{aligned}
$$




\section{Deformed aCtion of $\mathcal{H}$ on weight $\Pi(0)$-Modules and REalization of Verma MODULES}

As we noticed in Section 2, the free field realization from 2] does not provide realization of Verma modules $V^{\mathcal{H}}\left(h,(1-p) c_{L . I}\right)$ and their singular vectors in the case $p \geq 1$. In order to understand these Verma modules, we shall use certain deformation of free field realization from [2]. We shall use the construction from [4] to deform the action of the twisted Heisenberg-Virasoro algebra on $\Pi(0)$-modules (see also [8], [17]). Let

$$
\Delta(u, z)=z^{u_{0}} \exp \left(\sum_{n=1}^{\infty} \frac{u_{n}}{-n}(-z)^{-n}\right) .
$$

First we recall a definition of logarithmic modules. More information about structure theory of logarithmic modules can be found in literature on logarithmic vertex operator algebras (see [6], [15], 18], 22], 23] and reference therein).

Definition 5.1. (1) A module $\left(M, Y_{M}\right)$ for the conformal vertex algebra with conformal vector $\omega$ is a logarithmic module of rank $m \in \mathbb{Z}_{\geq 1}$ if

$$
\left(L(0)-L_{s s}(0)\right)^{m}=0, \quad\left(L(0)-L_{s s}(0)\right)^{m-1} \neq 0,
$$

where $L_{s s}(0)$ is the semisimple part of $L(0)$.

(2) If for every $m \in \mathbb{Z}_{\geq 1}\left(L(0)-L_{s s}(0)\right)^{m} \neq 0$ on $M$, we say that $\left(M, Y_{M}\right)$ is a logarithmic module of infinite rank.

Theorem 5.2. For every $\Pi(0)$-module $\left(M, Y_{M}(\cdot, z)\right)$,

$$
\left(\widetilde{M}, \widetilde{Y}_{\widetilde{M}}(\cdot, z)\right):=\left(M, Y_{M}(\Delta(u, z) \cdot, z)\right)
$$

is a $\overline{\Pi(0)}:=\operatorname{Ker}_{\Pi(0)} Q$-module. The action of Heisenberg-Virasoro algebra is

$$
\begin{aligned}
& \widetilde{I(z)}=\sum_{n \in \mathbb{Z}} \widetilde{I(n)} z^{-n-1}=\widetilde{Y}_{\widetilde{M}}(I, z)=I(z) \\
& \widetilde{L(z)}=\sum_{n \in \mathbb{Z}} \widetilde{L(n)} z^{-n-2}=\widetilde{Y}_{\widetilde{M}}(\omega, z)=L(z)+z^{-1} Y_{M}(u, z) .
\end{aligned}
$$

In particular,

$$
\widetilde{I(n)}=I(n), \quad \widetilde{L(n)}=L(n)+u_{n}
$$

and $\widetilde{L(0)}-\widetilde{L_{s s}(0)}=u_{0}=Q$.

Recall the definition of $\Pi(0)-$ modules

$$
\Pi(p, r):=\Pi(0) \cdot e^{\frac{p-1}{2} d^{2}+\frac{1-r}{2} c} \quad \text { where } \quad p \in \mathbb{Z}, r \in \mathbb{C} .
$$

Then $\widetilde{\Pi(p, r)}$ are logarithmic $\mathcal{H}$-modules which are uniquely determined by the action (5.19).

We shall also consider the cyclic submodules:

$$
\widetilde{W_{p, r}}=U(\mathcal{H}) \cdot e^{\frac{p-1}{2} d^{2}+\frac{1-r}{2} c} \subset \widetilde{\Pi(p, r)} .
$$


5.1. Case $h_{I}=(1-p) c_{L, I}$. We saw that for the undeformed action of $\mathcal{H}$ studied in [2], vector $v_{p, r}=e^{\frac{p-1}{2} d^{2}+\frac{1-r}{2} c}$, for $p \geq 1$, generates the irreducible highest weight module $W_{p, r}$. But we shall see below that $\widetilde{W_{p, r}}$ is isomorphic to a Verma module.

Theorem 5.3. Assume that $p \in \mathbb{Z}_{>0}, r \in \mathbb{C}$. We have

(1) $\widetilde{W_{p, r}} \cong V^{\mathcal{H}}\left(h_{p, r},(1-p) c_{L, I}\right)$.

(2) Singular vectors in $\widetilde{W_{p, r}} \cong V^{\mathcal{H}}\left(h_{p, r},(1-p) c_{L, I}\right)$ are

$$
\text { Sing }=\left\{v_{p, r-2 n} \mid n \geq 0\right\} \text {. }
$$

Proof. It is clear that $v_{p, r-2 n}=e^{\frac{p-1}{2} d^{2}+\frac{1-r}{2} c+n c}$ is a singular vector for any $n \geq 0$. We only need to prove that

$$
v_{p, r-2 n} \in \widetilde{W_{p, r}} \text {. }
$$

Assume that (5.20) holds for $n \in \mathbb{Z}_{\geq 0}$. Since $e_{k}^{c} v_{p, r-2 n}=0$ for every $k \geq 1-p$ we have

$$
\widetilde{L(k)}=L(k) \quad \text { on } \mathbb{C}[c(-1), c(-2), \ldots] \cdot v_{p, r-2 n} .
$$

But since $e_{-p}^{c} v_{p, r-2 n}=v_{p, r-2(n+1)}$ we have

$$
\widetilde{L(-p)} v_{p, r-2 n}=L(-p) v_{p, r-2 n}+v_{p, r-2(n+1)} .
$$

By using the expression for singular vector in $V^{\mathcal{H}}\left(h_{p, r},(1-p) c_{L, I}\right)$ from Proposition 4.2 we get for $h=h_{p, r-2 n}$

$$
\begin{aligned}
& \Phi_{p}(\widetilde{L}, c) \cdot v_{p, r-2 n} \\
= & \sum_{i=1}^{p}\left(\widetilde{L}(-i) S_{p-i}(-c)\right)+S_{p}(-c)\left(h+\frac{c_{L}-2}{24}(p-1)\right) \\
& \left.-\frac{c_{L}-26}{24}\left(\sum_{i=1}^{p}(i-1) c(-i) S_{p-i}(-c)\right)\right) v_{p, r-2 n} \\
= & \Phi_{p}(L, c) \cdot v_{p, r-2 n}+v_{p, r-2(n+1)} \\
= & v_{p, r-2(n+1)} .
\end{aligned}
$$

(Above we used the fact that $v_{p, r-2 n}$ generates the irreducible $\mathcal{H}$-module $L^{\mathcal{H}}\left(h_{p, r},(1-p) c_{L, I}\right)$ for the undeformed action, so $\Phi_{p}(L, c) \cdot v_{p, r-2 n}=0$.)

Thus we get that $v_{p, r-2(n+1)}=e^{\frac{p-1}{2} d^{2}+\frac{1-r}{2} c+(n+1) c}$ belongs to $\widetilde{W_{p, r}}$. The claim now follows by induction.

Finally, we obtain a deformed version of Theorem 3.2

Theorem 5.4. Let $Z^{(m)}=\operatorname{Ker} \widetilde{\Pi(p, r)} Q^{m+1}$. Then we have

(1) $\widetilde{\Pi(p, r)} \cong \bigcup_{m \geq 0} Z^{(m)}, \quad \Pi(0)^{(n)} \cdot Z^{(m)} \subset Z^{(n+m)}$.

(2) For every $m \in \mathbb{Z}_{\geq 0}, Z^{(m)}$ is a logarithmic $\overline{\Pi(0)}$-module of rank $m+1$ with respect to $\widetilde{L(0)}$.

(3) $Z^{(m)} / Z^{(m-1)}$ is a weight $\overline{\Pi(0)}$-module which is as $\mathcal{H}$-module isomorphic to

$$
\bigcup_{n \in \mathbb{Z}} \widetilde{W_{p, r-2 n}}
$$


Proof. Assertion (1) is clear. Using relation (2.13) in the proof of Lemma 2.2 we see that $v_{p, r-2 \ell}^{(m)} \in Z^{(m)} \backslash Z^{(m-1)}$. Since $\widetilde{L(0)}-\widehat{L_{s s}(0)}=Q$, we have that $Z^{(m)}$ is a logarithmic module of $\widetilde{L(0)}-$ nilpotent rank $m+1$ so (2) holds. Assertion (3) results from following facts:

(a) As an $\mathcal{H}$-module $\widetilde{\Pi(p, r)}$ is generated by set of vectors

$$
\left\{v_{p, r-2 \ell} \mid \ell \in \mathbb{Z}\right\} \bigcup\left\{v_{p, r-2 \ell}^{(m)}, \mid m, \ell \in \mathbb{Z}, m \geq 1\right\} .
$$

(b) $Z^{(m)} / Z^{(m-1)}$ is a weight $\mathcal{H}$-module (i.e., non-logarithmic) generated by vectors $\left\{v_{p, r-2 j}^{(m)}+\right.$ $\left.Z^{(m-1)} \mid \ell \in \mathbb{Z}\right\}$.

(c) $v_{p, r-2 \ell}^{(m)}+Z^{(m-1)}$ generates the Verma module $\widetilde{W_{p, r-2 \ell}}$.

Since $Q$ and $e_{-j}^{c}$ commute and by using (2.12) we get

$$
Q^{m} e_{-j}^{c} v_{p, r-2 \ell}^{(m)}=e_{-j}^{c} v_{p, r-2(\ell+m)}=S_{j-p}(c) v_{p, r-2(\ell+m)}
$$

so $e_{-j}^{c} v_{p, r-2 \ell}^{(m)} \in Z^{(m-1)}$ for $j<p$. Therefore

$$
\widetilde{L(-j)} v_{p, r-2 \ell}^{(m)}= \begin{cases}L(-j) v_{p, r-2 \ell}^{(m)} \bmod Z^{(m-1)}, & j<p, \\ L(-p) v_{p, r-2 \ell}^{(m)}+v_{p, r-2(\ell+1)}^{(m)}, & j=p .\end{cases}
$$

The proof of claims (a) and (b) easily follows from Theorem 3.2 and (5.22). Let us prove claim (c).

We have proved in (5.22) that $v_{p, r-2 \ell}^{(m)}+Z^{(m-1)}$ is a highest weight vector with highest weight $\left(h_{p, r-2 \ell},(1-p) c_{L, I}\right)$. Now, repeating the same arguments as in the proof of Theorem 5.3 we get

$$
\begin{aligned}
& \Phi_{p}(\widetilde{L}, c) \cdot v_{p, r-2 \ell}^{(m)} \\
=\quad & \Phi_{p}(L, c) \cdot v_{p, r-2 \ell}^{(m)}+v_{p, r-2(\ell+1)}^{(m)} \bmod Z^{(m-1)} \\
=\quad & v_{p, r-2(\ell+1)}^{(m)} \bmod Z^{(m-1)} .
\end{aligned}
$$

This implies that $v_{p, r-2 \ell}^{(m)}+Z^{(m-1)}$ generates the Verma module $\widetilde{W_{p, r-2 \ell}}$ which contains all Verma modules $W_{p, r-2(\ell+j)}, j \in \mathbb{Z}_{\geq 1}$. This completes the proof. (See also Figure 3 in Appendix $\mathrm{A}$ where one can follow steps in the proof.)

5.2. Case $h_{I}=c_{L, I}$. Note that $\widetilde{\Pi(0, r)}$ is an $\mathcal{H}$-module on which $I(0)$ acts as multiplication by $c_{L, I}$.

In particular, $\widetilde{W_{0, r}}$ is a $\mathbb{Z}_{\geq 0}$-graded logarithmic $\mathcal{H}$-module whose lowest component is

$$
\widetilde{W_{0, r}}(0):=\operatorname{span}_{\mathbb{C}}\left\{v_{0, r-2 \ell} \mid \ell \in \mathbb{Z}_{\geq 0}\right\}
$$

Moreover, since

$$
\widetilde{L(0)}-\frac{c_{L}-2}{24}=Q, \quad Q^{n} v_{0, r}=v_{0, r-2 n}
$$

we conclude that $\widetilde{W_{0, r}}$ is a $\mathbb{Z}_{\geq 0^{-}}$graded logarithmic module of infinite rank. See Figure 4 in Appendix A. 
5.3. Case $h_{I}=(1+p) c_{L, I}$. We saw that $\Pi(-p, r)$ contains a descending chain of submodules $\Pi(-p, r)^{(m)}$ isomorphic to $\Pi(-p, r)$ (Theorem 3.3$)$.

Theorem 5.5. Let $p \in \mathbb{Z}_{>0}$. Then $\widetilde{\Pi(-p, r)}$ is a logarithmic $\overline{\Pi(0)}$-module such that

$$
\operatorname{dim} \mathbb{C}[\widetilde{L(0)}] v=\infty \quad \text { for every } v \in \widetilde{\Pi(-p, r)}
$$

Quotient $\widetilde{\Pi(-p, r)} / \widetilde{\Pi(-p, r)}$ is a weight module such that

$$
\widetilde{L(n)} v_{-p, r}=S_{p-n}(c) v_{-p, r-2} \quad(1 \leq n \leq p) \quad \text { and } \widetilde{L(n)} v_{-p, r}=0 \quad(n>p) .
$$

Proof. Let $S=\left\{v_{-p, r-2 \ell} \mid \ell \in \mathbb{Z}\right\}$. Let $\langle S\rangle$ be the $\mathcal{H}$-submodule generated by the set $S$. We shall first prove that $\Pi \widetilde{(-p, r)}=\langle S\rangle$. Since, as a vector space $\Pi \widetilde{(-p, r)} \cong \Pi(-p, r) \cong$ $\bigoplus_{\ell \in \mathbb{Z}} W_{-p, r-2 \ell}$, it suffices to show that $W_{-p, r-2 \ell} \subset\langle S\rangle$ for each $\ell$.

Take an arbitrary basis vector

$$
u=c\left(-p_{1}\right) \cdots c\left(-p_{s}\right) L\left(-q_{1}\right) \cdots L\left(-q_{m}\right) v_{-p, r-2 \ell}
$$

of $W_{-p, r-2 \ell}$, where $\ell \in \mathbb{Z}, p_{1}, \ldots, p_{s}, q_{1}, \ldots, q_{m} \geq 1$. Then by applying formula for $\widetilde{L(n)}$ and relation $\left[e_{m}^{c}, L(n)\right]=m e_{m+n}^{c}$ we get

$$
\begin{aligned}
& c\left(-p_{1}\right) \cdots c\left(-p_{s}\right) \widetilde{L\left(-q_{1}\right)} \cdots \widetilde{L\left(-q_{m}\right)} v_{-p, r-2 \ell} \\
= & c\left(-p_{1}\right) \cdots c\left(-p_{s}\right) L\left(-q_{1}\right) \cdots L\left(-q_{m}\right) v_{-p, r-2 \ell}+w
\end{aligned}
$$

where $w$ is a linear combination of vectors

$$
c\left(-t_{1}\right) \cdots c\left(-t_{s^{\prime}}\right) L\left(-u_{1}\right) \cdots L\left(-u_{m^{\prime}}\right) v_{-p, r-2 \ell^{\prime}}
$$

such that $\ell^{\prime} \in \mathbb{Z}, m^{\prime}<m$. The assertion now follows by induction on $m$.

Furthermore, we have

$$
(\widetilde{L(0)}-L(0))^{n} v_{-p, r}=Q^{n} v_{-p, r}=\left(S_{p}(c)\right)^{n} v_{-p, r-2 n} .
$$

Since $Q$ commutes with action of $\mathcal{H}$, we proved the first claim.

Taking a quotient by $\widetilde{\Pi(-p, r)}{ }^{(1)}=\operatorname{Im} Q$ results in a weight module (i.e., non logarithmic module) on which $\widetilde{L(0)} \equiv L(0)$.

See Figure 5 in Appendix $\mathrm{A}$.

Remark 5.6. As far as we know, modules $\widetilde{\Pi(-p, r)} / \widetilde{\Pi(-p, r)}{ }^{(1)}$, and their cyclic submodules generated by images of $v_{-p, r-2 \ell}$ are weight $\mathcal{H}$-modules which haven't been analysed in the literature.

\section{Realization of Self-Dual modules via Whittaker П(0)-Modules}

In Section 5 we slightly refined the free field realization from [2], but these results still don't give a realization of all irreducible self-dual modules. In order to construct all self-dual modules we shall apply the deformation from Section 5 on the Whittaker $\Pi(0)$-module $\Pi_{\lambda}$ which was constructed in [7, Section 11] and used for a realization of Whittaker $A_{1}^{(1)}$-modules at the critical level. As a by-product we shall see that self-dual modules for $\mathcal{H}$ have non-trivial self-extensions which are logarithmic modules. 
6.1. Whittaker $\Pi(0)-$ module $\Pi_{\lambda}$. We shall recall the construction of a Whittaker $\Pi(0)-$ module $\Pi_{\lambda}$ from [7, Section 11]. Let $u=e^{c}, u^{-1}=e^{-c}$.

Theorem 6.1. [7] Assume that $\lambda \neq 0$. There is a $\Pi(0)$-module $\Pi_{\lambda}$ generated by the cyclic vector $w_{\lambda}$ such that $c(0)=-I d$ on $\Pi_{\lambda}$ and

$$
u_{0} w_{\lambda}=\lambda w_{\lambda}, \quad u_{0}^{-1} w_{\lambda}=\frac{1}{\lambda} w_{\lambda}, \quad u_{n} w_{\lambda}=u_{n}^{-1} w_{\lambda}=0(n \geq 1) .
$$

As a vector space

$$
\Pi_{\lambda} \cong \mathbb{C}[d(-n), c(-n-1) \mid n \geq 0]=\mathbb{C}[d(0)] \otimes M(1) .
$$

$\Pi_{\lambda}$ is $\mathbb{Z}_{\geq 0}$-graded

$$
\Pi_{\lambda}=\bigoplus_{n \in \mathbb{Z}_{\geq 0}} \Pi_{\lambda}(n)
$$

and lowest component is isomorphic to $\mathbb{C}[d(0)]$.

Recall also (cf. [7]) that the lowest component $\Pi_{\lambda}(0)$ is an irreducible Whittaker module for the associative algebra $\mathcal{A}$ defined by generators

$$
d(0), e^{n c} \quad(n \in \mathbb{Z})
$$

and relations

$$
\left[d(0), e^{n c}\right]=2 n e^{n c}, e^{n c} e^{m c}=e^{(n+m) c} \quad(n, m \in \mathbb{Z}) .
$$

6.2. Realization of self-dual modules. Now we can apply Theorem 5.2 on the Whittaker $\Pi(0)$-module $\Pi_{\lambda}$. We get $\mathcal{H}$-module $\widetilde{\Pi}_{\lambda}$, which is as a vector space isomorphic to $\Pi_{\lambda}$ and the (deformed) action of $\mathcal{H}$ is as follows:

$$
\widetilde{I(0)} \equiv-c_{L, I} c(0) \equiv c_{L, I} \mathrm{Id} \quad \text { on } \widetilde{\Pi}_{\lambda},
$$

and on the lowest component $\widetilde{\Pi}_{\lambda}(0)$ we have

$$
\begin{aligned}
\widetilde{L(0)} & \equiv \frac{1}{2} d(0)(c(0)+1)-\frac{c_{L}-2}{24} c(0)+u_{0} \quad \text { on } \widetilde{\Pi}_{\lambda}(0) \\
& \equiv \frac{c_{L}-2}{24} \mathrm{Id}+u_{0} \quad \text { on } \Pi_{\lambda}(0)
\end{aligned}
$$

This implies:

$$
\widetilde{I(0)} w_{\lambda}=c_{L, I} w_{\lambda}, \quad \widetilde{L(0)} w_{\lambda}=\left(\frac{c_{L}-2}{24}+\lambda\right) w_{\lambda} .
$$

Define also the following (logarithmic) cyclic module:

$$
\widetilde{\Pi}_{\lambda}^{(n)}=U(\mathcal{H}) \cdot d(0)^{n} w_{\lambda} .
$$

Lemma 6.2. We have:

$$
\widetilde{\Pi}_{\lambda}^{(n+1)} \supset \widetilde{\Pi}_{\lambda}^{(n)}, \quad \widetilde{\Pi}_{\lambda}=\bigcup_{n \in \mathbb{Z}_{\geq 0}} \widetilde{\Pi}_{\lambda}^{(n)} .
$$

Proof. By using (6.24) one can easily see that for $0 \leq m \leq n$ there is a polynomial $P(x)$ such that

$$
P(\widetilde{L(0)}) d(0)^{n} w_{\lambda}=d(0)^{m} w_{\lambda} .
$$

This proves that $\widetilde{\Pi}_{\lambda}^{(n+1)} \supset \widetilde{\Pi}_{\lambda}^{(n)}$ for $n \in \mathbb{Z}_{\geq 0}$. 
Take an arbitrary basis vector

$$
u=c\left(-p_{1}\right) \cdots c\left(-p_{s}\right) d\left(-q_{1}\right) \cdots d\left(-q_{r}\right) d(0)^{\ell} w_{\lambda}
$$

of $\widetilde{\Pi}_{\lambda}$, where $\ell \in \mathbb{Z}_{\geq 0}, p_{1}, \ldots, p_{s}, q_{1}, \ldots, q_{r} \geq 1$. Then

$$
\begin{aligned}
& c\left(-p_{1}\right) \cdots c\left(-p_{s}\right) \widetilde{L\left(-q_{1}\right)} \cdots \widetilde{L\left(-q_{r}\right)} d(0)^{\ell} w_{\lambda} \\
= & A c\left(-p_{1}\right) \cdots c\left(-p_{s}\right) d\left(-q_{1}\right) \cdots d\left(-q_{r}\right) d(0)^{\ell} w_{\lambda}+w
\end{aligned}
$$

where $A \neq 0$ and $w$ is a linear combination of vectors

$$
c\left(-t_{1}\right) \cdots c\left(-t_{s^{\prime}}\right) d\left(-u_{1}\right) \cdots d\left(-u_{r^{\prime}}\right) d(0)^{\ell^{\prime}} w_{\lambda}
$$

such that $\ell^{\prime} \in \mathbb{Z}_{\geq 0}, r^{\prime}<r$ or $r=r^{\prime}$ and $u_{1}+\cdots+u_{r^{\prime}}<q_{1}+\cdots+q_{r}$. The assertion now follows by induction.

Theorem 6.3. For every $\lambda \in \mathbb{C}, \lambda \neq 0$ we have:

(1) $\widetilde{\Pi}_{\lambda}$ is a logarithmic $\mathcal{H}$-module of infinite rank with respect to $\widetilde{L(0)}$.

(2) $\widetilde{\Pi}_{\lambda}^{(0)}$ is an irreducible self-dual $\mathcal{H}$-module with highest weight

$$
\left(h, h_{I}\right)=\left(\frac{c_{L}-2}{24}+\lambda, c_{L, I}\right) .
$$

(3) $\mathcal{H}$-module $\widetilde{\Pi}_{\lambda}$ admits the $\mathbb{Z}_{\geq 0}$-filtration:

$$
\widetilde{\Pi}_{\lambda}=\cup_{n \in \mathbb{Z}_{\geq 0}} \widetilde{\Pi}_{\lambda}^{(n)}
$$

such that

$$
\widetilde{\Pi}_{\lambda}^{(0)}=L^{\mathcal{H}}\left(h, h_{I}\right), \quad \widetilde{\Pi}_{\lambda}^{(n+1)} / \widetilde{\Pi}_{\lambda}^{(n)} \cong L^{\mathcal{H}}\left(h, h_{I}\right) .
$$

Every $\widetilde{\Pi}_{\lambda}^{(n)}$ is a logarithmic $\mathcal{H}$-module of rank $n$ with respect to $\widetilde{L(0)}$.

Proof. (1) follows from the fact that on the top component $\widetilde{\Pi}_{\lambda}(0)$ we have $Q=u_{0}=\widetilde{L(0)}-$ $\frac{c_{L}-2}{24}$.

(2) Using (6.25) and the fact that the Verma module with highest weight $\left(h, h_{I}\right)=\left(\frac{c_{L}-2}{24}+\right.$ $\left.\lambda, c_{L, I}\right)$ is irreducible we get $L^{\mathcal{H}}\left(h, h_{I}\right)=U(\mathcal{H}) \cdot w_{\lambda}=\widetilde{\Pi}_{\lambda}^{(0)}$.

(3) First we notice that for $m \geq 0$ we have

$$
\widetilde{L(m)} d(0)^{n+1} w_{\lambda}=h \delta_{m, 0} d(0)^{n+1} w_{\lambda} \bmod \widetilde{\Pi}_{\lambda}^{(n)} .
$$

Therefore we have isomorphism $L^{\mathcal{H}}\left(h, h_{I}\right) \rightarrow \widetilde{\Pi}_{\lambda}^{(n+1)} / \widetilde{\Pi}_{\lambda}^{(n)}$. The proof now follows from Lemma 6.2.

See Figure 6 in Appendix $\mathrm{A}$ for reference.

We list two interesting consequences of previous theorem.

Corollary 6.4. Logarithmic $\mathcal{H}$-module $\widetilde{\Pi}_{\lambda}^{(1)}$ is a non-split self-extension of irreducible selfdual module $L^{\mathcal{H}}\left(h, h_{I}\right)$ :

$$
0 \rightarrow L^{\mathcal{H}}\left(h, h_{I}\right) \rightarrow \widetilde{\Pi}_{\lambda}^{(1)} \rightarrow L^{\mathcal{H}}\left(h, h_{I}\right) \rightarrow 0 .
$$

Note that the vertex algebra $\overline{\Pi(0)}$ is not $\mathbb{Z}_{\geq 0}$-graded since for every $n \in \mathbb{Z}, e^{n c}$ has weight $n$. Irreducible $\overline{\Pi(0)}$-modules from Theorem 5.4 (3) are not $\mathbb{Z}_{\geq 0}$-graded. But, quite surprisingly, the vertex algebra $\overline{\Pi(0)}$ admits a large family of $\mathbb{Z}_{\geq 0}$-graded modules which are self-dual. We also construct a family of intertwining operators which haven't appeared in [2]. 
Corollary 6.5. We have:

(1) $L^{\mathcal{H}}\left(h, c_{L, I}\right)$ is an irreducible $\mathbb{Z}_{\geq 0}$-graded $\overline{\Pi(0)}$-module.

(2) For every $n \in \mathbb{Z}$ there is a non-trivial intertwining operator of type

$$
\left(\begin{array}{c}
L^{\mathcal{H}}\left(h, c_{L, I}\right) \\
L^{\mathcal{H}}(n, 0) \quad L^{\mathcal{H}}\left(h, c_{L, I}\right)
\end{array}\right)
$$

7. Some applichtions to the $W(2,2)$-Algebra

In [2] we introduced a free field realization of the $W(2,2)$-algebra as a subalgebra of the Heisenberg Virasoro algebra.

Recall that $W(2,2)$ is realized as a subalgebra of $L^{\mathcal{H}}\left(c_{L}, c_{L, I}\right)$ generated by $L(z)$ and

$$
W(z)=c_{L, I}^{2} \bar{W}(z)
$$

where

$$
\bar{W}(z)=\sum_{n \in \mathbb{Z}} \bar{W}(n) z^{-n-2}=Y\left(c(-1)^{2}-2 c(-2), z\right)=c(z)^{2}-2 \partial c(z) .
$$

In the paper [3] we discussed a free field realization of highest weight $W(2,2)$-modules. We constructed in [3, Section 4] $W(2,2)$-homomorphism $S_{1}: L^{\mathcal{H}}\left(c_{L}, c_{L, I}\right) \rightarrow L^{\mathcal{H}}(1,0)$ such that $\operatorname{Ker}_{L^{\mathcal{H}}\left(c_{L}, c_{L, I}\right)} S_{1}$ is the simple vertex algebra $L^{W(2,2)}\left(c_{L},-24 c_{L, I}^{2}\right)$. In this paper shall present a bosonic, non-local expression for the screening operator $S_{1}$.

The vertex algebra $W(2,2)$ has appeared in physics literature as the Galilean Virasoro algebra ([25], 9], [10]) and as $\mathrm{BMS}_{3}$ algebra ([11]). We noticed a free field realization of the $W(2,2)$-algebra in terms of the $\beta \gamma$ systems in [11]. We shall see how this realization relates to our approach.

7.1. A bosonic formula for the second screening operator and $W(2,2)$-algebra. Our approach is motived by the realization of screening operators in LCFT from [5] and [6]. Recall the definition of modules $W_{p, r}$ from (2.11). For $r \in \mathbb{Z}$ we define:

$$
\begin{aligned}
S & =-\operatorname{Res}_{z} \operatorname{Res}_{z_{1}}\left(\log \left(1-\frac{z_{1}}{z}\right) e^{c}(z) d^{1}\left(z_{1}\right)-\log \left(1-\frac{z}{z_{1}}\right) d^{1}\left(z_{1}\right) e^{c}(z)\right) \\
& =\sum_{j=1}^{\infty} \frac{1}{j}\left(d^{1}(-j) e_{j}^{c}-e_{-j}^{c} d^{1}(j)\right): W_{p, r} \rightarrow W_{p, r-2} .
\end{aligned}
$$

Lemma 7.1. We have:

$$
\begin{aligned}
& {[L(m), S]=d^{1}(m) e_{0}^{c}-e_{m}^{c} d^{1}(0)+2 \delta_{m, 0} e_{0}^{c},} \\
& {[c(m), S]=2 e_{m}^{c}-2 \delta_{m, 0} e_{0}^{c},[W(m), S]=0 .}
\end{aligned}
$$

Proof. In the proof we use the following formulas

$$
\begin{aligned}
{\left[L(n), e_{m}^{c}\right] } & =-m e_{n+m}^{c}, \\
{\left[L(n), d^{1}(m)\right] } & =-m d^{1}(n+m)-2\left(m^{2}-m\right) \delta_{m+n, 0} .
\end{aligned}
$$


We have:

$$
\begin{aligned}
{[L(n), S]=} & \sum_{j=1}^{\infty} \frac{-j}{j}\left(d^{1}(-j) e_{j+n}^{c}+e_{-j+n}^{c} d^{1}(j)\right) \\
& +\sum_{j=1}^{\infty} \frac{j}{j}\left(d^{1}(-j+n) e_{j+n}^{c}+e_{-j}^{c} d^{1}(j+n)\right) \\
& -2 \sum_{j=1}^{\infty} \frac{j^{2}+j}{j} \delta_{-j+n, 0} e_{j+n}^{c}+2 \sum_{j=1}^{\infty} \frac{j^{2}-j}{j} \delta_{j+n, 0} e_{-j}^{c} \\
= & -\sum_{j=1}^{\infty}\left(d^{1}(-j) e_{j+n}^{c}+e_{-j+n}^{c} d^{1}(j)\right)+\sum_{j=1}^{\infty}\left(d^{1}(-j+n) e_{j}^{c}+e_{-j}^{c} d^{1}(j+n)\right) \\
& -2 \sum_{j=1}^{\infty}(j+1) \delta_{-j+n, 0} e_{j}^{c}+2 \sum_{j=1}^{\infty}(j-1) \delta_{j+n, 0} e_{-j}^{c} \\
= & -2(n+1) e_{n}^{c}+2 \delta_{n, 0} e_{0}^{c}+d^{1}(0) e_{n}^{c}+\cdots+d^{1}(n-1) e_{1}^{c} \\
& -\left(e_{n-1}^{c} d^{1}(1)+\cdots+e_{1}^{c} d^{1}(n-1)\right)-e_{0}^{c} d^{1}(n) \\
= & -d^{1}(n) e_{0}^{c}+e_{n}^{c} d^{1}(0)+2 \delta_{n, 0} e_{0}^{c} .
\end{aligned}
$$

Relation $[c(m), S]=2\left(1-\delta_{m, 0}\right) e_{m}^{c}$ follows directly from the definition of the operator $S$. Next we have

$$
\begin{aligned}
{[\bar{W}(n), S] } & =\left(\sum_{k \in \mathbb{Z}}[c(k) c(n-k), S]\right)+2 n[c(n-1), S] \\
& =-\sum_{k \in \mathbb{Z}}\left(c(k) e_{n-k}^{c}+c(n-k) e_{k}^{c}\right)-2 n e_{n-1}^{c} \\
& =-4\left(D e^{c}\right)_{n}-4 n e_{n-1}^{c}=0
\end{aligned}
$$

The proof follows.

Now, we will see that in the case $r=1$ our operator $S$ is a multiple of the screening operator $S_{1}$ from 3$]$ :

Corollary 7.2. Let $r=1$, and consider $S: W_{p, 1} \rightarrow W_{p,-1}$. Then $S$ commutes with the action of the $W(2,2)$-algebra:

$$
[S, W(n)]=[S, L(n)]=0 \quad(n \in \mathbb{Z}) .
$$

Moreover, $S$ is a $W(2,2)$-homomorphism which is proportional to $S_{1}$.

Proof. In the case $r=1$ we have that $d^{1}(0)$ and $Q=e_{0}^{c}$ act trivially on $W_{p, 1}$, and therefore Lemma 7.1 implies that

$$
[S, W(n)]=[S, L(n)]=0 \quad(n \in \mathbb{Z}) .
$$

It is clear that the $W(2,2)$-homomorphism $S_{1}: W_{p, 1} \rightarrow W_{p,-1}$ from [3, Section 4] is uniquely determined by the properties

$$
\left[S_{1}, L(n)\right]=0, \quad\left[S_{1}, I(n)\right]=-e_{n}^{c},
$$

which gives $\left[S_{1}, c(n)\right]=\frac{1}{c_{L, I}} e_{n}^{c}$. Now Lemma 7.1 gives that $S=2 c_{L, I} S_{1}$. 
7.2. On the Banerjee, Jatkar, Mukhi, Neogi's free field realization of the BMS $_{3}-$ algebra. Recently, Banerjee, Jatkar, Mukhi and Neogi in [11] have discovered a new free field realization of the $W(2,2)$-algebra for central charge $c_{L}=26$. The vertex algebra $L^{W(2,2)}\left(26, c_{W}\right)$ is realized inside of the $\beta \gamma$ system. Since the $\beta \gamma$-system can be embedded into the vertex algebra $\Pi(0)$, one may try to extend this realization in order to obtain an arbitrary central charge $c_{L}$. Quite surprisingly, even in the case of the larger vertex algebra $\Pi(0)$, one gets the $W(2,2)$-structure only for $c_{L}=26$.

Recall the definition of following Virasoro vector of central charge $c_{L} \in \mathbb{C}$.

$$
\omega=\frac{1}{2} c(-1) d(-1)+\frac{c_{L}-2}{24} c(-2)-\frac{1}{2} d(-2) .
$$

We shall now deform this vector in a different way:

Lemma 7.3. For every $\mu \in \mathbb{C}$

$$
\widetilde{\omega}=\omega+\mu e_{-4}^{-c} \mathbf{1}=\omega+\frac{\mu}{6} D^{3} e^{-c} .
$$

is a Virasoro vector of central charge $c_{L}$.

The proof of lemma follows from a more general statement (which is also noticed in [11]):

Claim: Assume that $(V, Y, \mathbf{1}, \omega)$ is a VOA of central charge $c$, and $v$ is a primary, commutative vector of conformal weight -1 . Then $\widetilde{\omega}=\omega+\frac{1}{6} D^{3} v$ is a Virasoro vector of central charge $c$.

The following result is obtained in [11, Section 2]. We include a proof of this result from which one can see that such construction works only for $c_{L}=26$.

Proposition 7.4. 11]. The vertex algebra $L^{W(2,2)}\left(c_{L}, c_{W}\right)$ for $c_{L}=26$ is isomorphic to a vertex subalgebra of $\Pi(0)$ generated by

$$
\begin{aligned}
\widetilde{\omega} & =\frac{1}{2} c(-1) d(-1)+\frac{c_{L}-2}{24} c(-2)-\frac{1}{2} d(-2)+\frac{\mu}{6} D^{3} e^{-c} \\
w & =\left(d(-1)+\frac{c_{L}-14}{12} c(-1)\right) e^{c}
\end{aligned}
$$

where

$$
\mu=-\frac{c_{W}}{4}
$$

Proof. By direct calculation we get

$$
\begin{aligned}
w_{0} w & =\frac{c_{L}-26}{3} c(-1) e^{2 c}=\frac{c_{L}-26}{6} D e^{2 c} \\
w_{1} w & =\frac{c_{L}-26}{3} e^{2 c} \\
w_{n} w & =0 \quad(n \geq 2) .
\end{aligned}
$$

By using formulas

$$
\begin{aligned}
{[L(n), c(m)] } & =-m c(n+m)+\left(m^{2}-m\right) \delta_{m+n, 0} \\
{[L(n), d(m)] } & =-m d(n+m)-\frac{c_{L}-2}{12}\left(m^{2}-m\right) \delta_{m+n, 0}
\end{aligned}
$$


we get that

$$
\begin{aligned}
L(1) w & =\left([L(1), d(-1)]+\frac{c_{L}-14}{12}[L(1), c(-1)]\right) e^{c} \\
& =\left(2-\frac{c_{L}-2}{6}+\frac{c_{L}-14}{6}\right) e^{c} \\
& =0
\end{aligned}
$$

which easily implies that

$$
L(n) w=2 \delta_{n, 0} w \quad(n \geq 0) .
$$

Since

$$
\widetilde{L}(2) w=-\mu e_{0}^{-c} w=-2 \mu=\frac{c_{W}}{2},
$$

we get

$$
\widetilde{L}(n) w=2 \delta_{n, 0} w+\frac{c_{W}}{2} \delta_{n, 2} \quad(n \geq 0) .
$$

Claim now follows from (7.30)-(7.32), (77.36) and Lemma 7.3.

Remark 7.5. The Weyl vertex algebra (also called the $\beta \gamma$ system in the physical literature) can be realized as a subalgebra of the vertex algebra $\Pi(0)$ as follows:

$$
\beta=(c(-1)+d(-1)) e^{c}, \gamma=-\frac{1}{2} e^{-c} .
$$

Then the components of the fields $Y(\beta, z)=\sum_{n \mathbb{Z}} \beta(n) z^{-n-1}, Y(\gamma, z)=\sum_{n \in \mathbb{Z}} \gamma(n) z^{-n}$ satisfy the commutation relation for the Weyl algebra

$$
[\beta(n), \beta(m)]=[\gamma(n), \gamma(m)]=0, \quad[\beta(n), \gamma(m)]=\delta_{n+m, 0} .
$$

The vertex algebra $\Pi(0)$ can be treated as a certain localization of the Weyl vertex algebra (for details see [16], 21], 7]).

By using 7.37) we see that for $c_{L}=26 L^{W(2,2)}\left(c_{L}, c_{W}\right)$ is realized as a subalgebra of the Weyl vertex algebra, which corresponds to the realization in [11].

Remark 7.6. It would be interesting to investigate the structure of $W(2,2)$-modules $\Pi(p, r)$ with this new action. Since

$$
\widetilde{L(n)}=L(n)-\frac{1}{6}(n+1) n(n-1) \mu e_{n-2}^{-c},
$$

we have that $\Pi(p, r)$ and $\Pi_{\lambda}$ are weight $L^{W(2,2)}\left(c_{L}, c_{W}\right)-$ modules on which $\widetilde{L(0)}=L(0)$. In our forthcoming papers we plan to investigate the appearance of these modules in the fusion rules analysis at $c_{L}=26$.

\section{Appendix A. Figures}

Here we present some visualizations of modules $\Pi(p, r)$ and $\widetilde{\Pi(p, r)}$. 


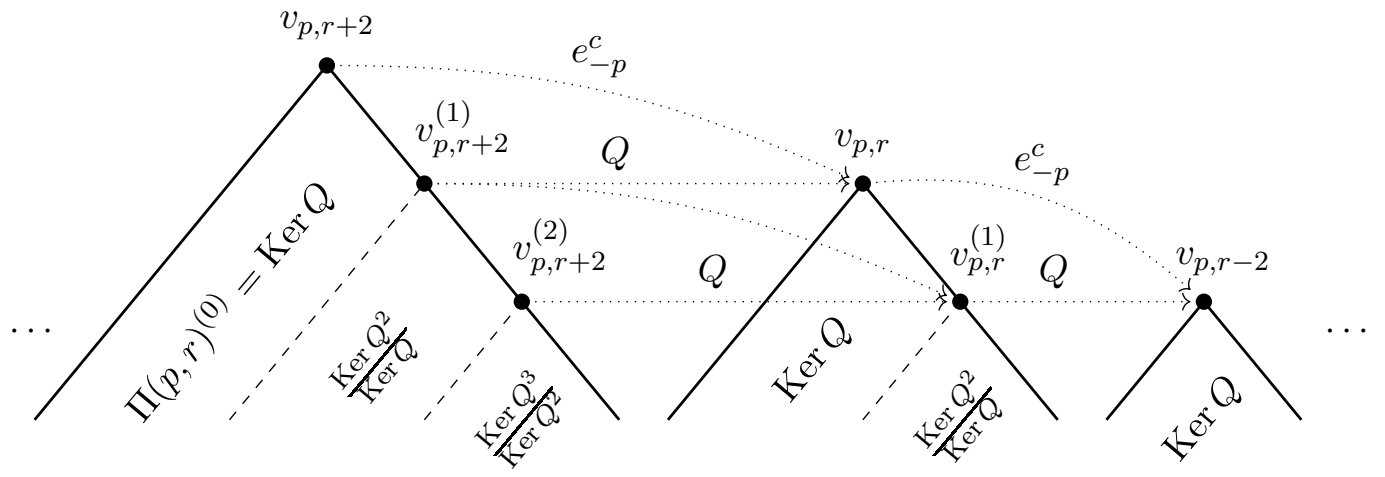

Figure 1. $\overline{\Pi(0)}$-module $\Pi(p, r), p \in \mathbb{Z}_{>0}$

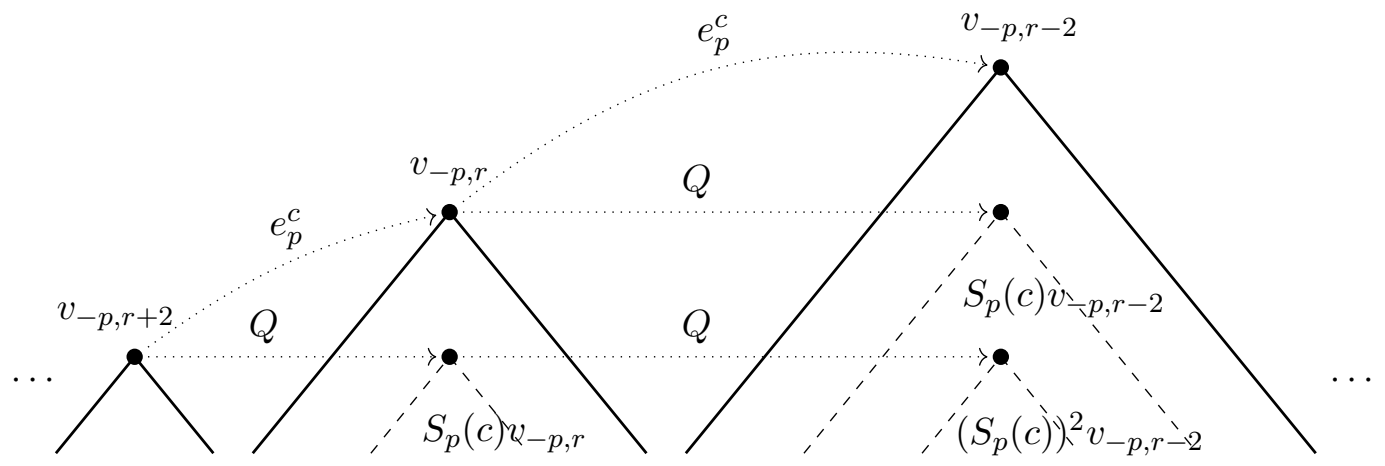

Figure 2. $\overline{\Pi(0)}$-module $\Pi(-p, r), p \in \mathbb{Z}_{>0}$

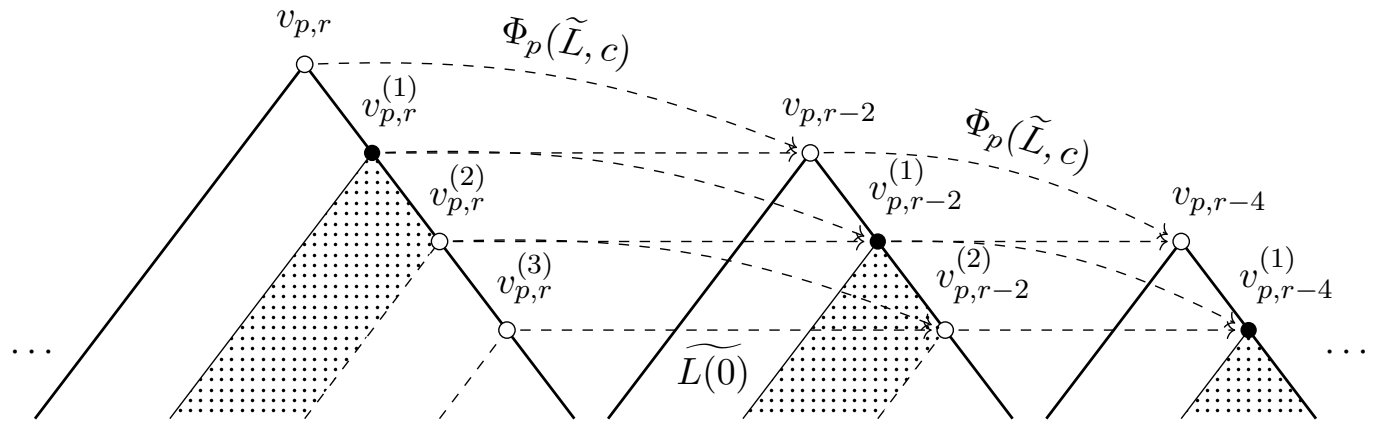

Figure 3. Deformed action of $\mathcal{H}$ on $\widetilde{\Pi(p, r)}, p>0$. Dotted area represents a cyclic submodule of $\Pi \widetilde{(p, r)^{(1)}} / \widetilde{\Pi(p, r)^{(0)}}$ generated by $v_{p, r}^{(1)}$ which is isomorphic to $\widetilde{W_{p, r-2}} \cong V^{\mathcal{H}}\left(h_{p, r}+p, h_{I}\right)$. Arrows represent $\Phi_{p}(\widetilde{L}, c)$ (descending), and $\widetilde{L(0)}$ (horizontal). 


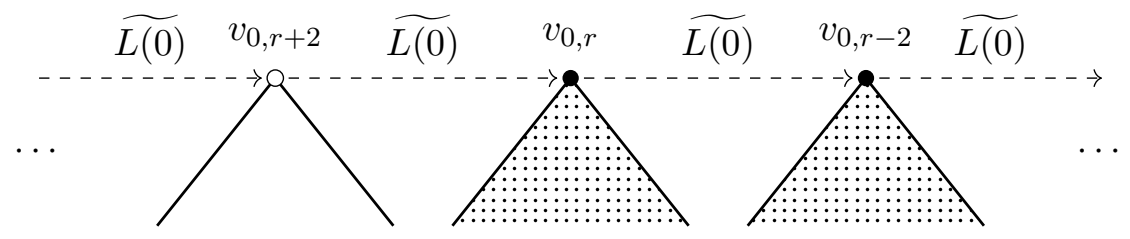

Figure 4. $\overline{\Pi(0)}$-module $\widetilde{\Pi(0, r)}$. Dotted area represents a portion of a deformed $\mathcal{H}$-module $\widetilde{W_{0, r}}$.

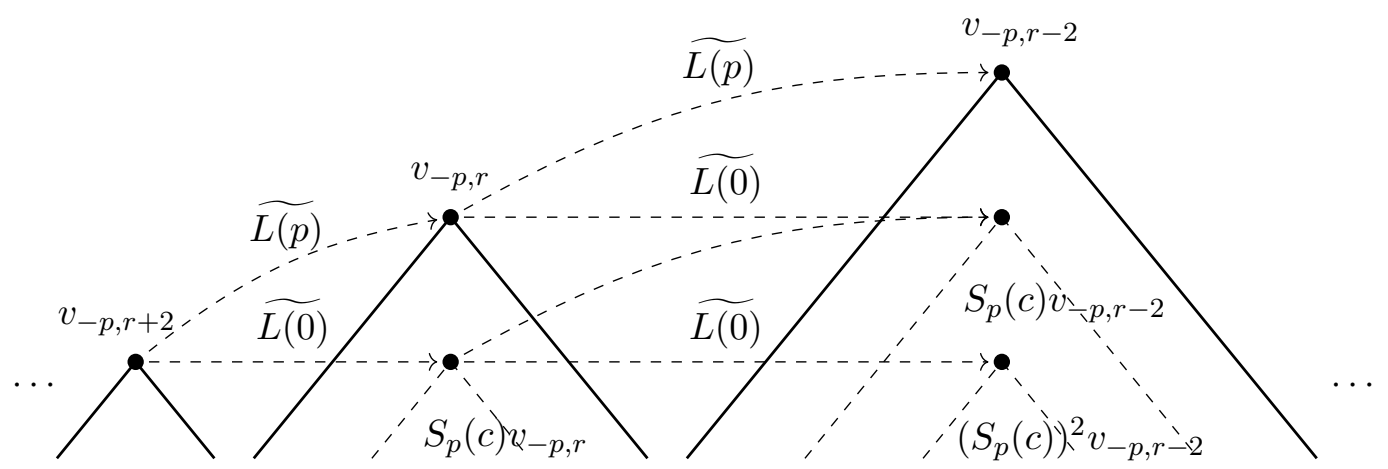

Figure 5. Deformed action of $\mathcal{H}$ on $\widetilde{\Pi(-p, r)}, p \in \mathbb{Z}_{>0}$

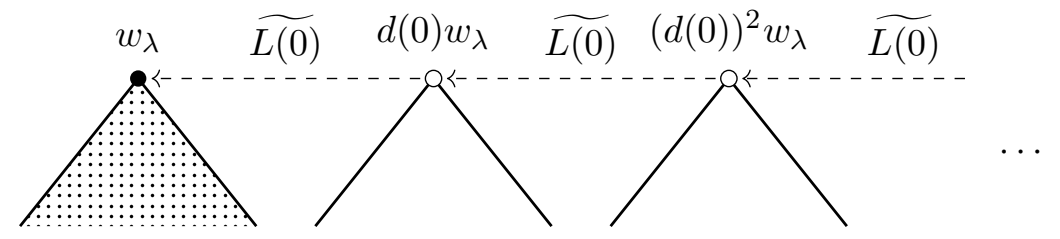

Figure 6. A deformed Whittaker module $\widetilde{\Pi}_{\lambda}$. Dotted area represents $\widetilde{\Pi}_{\lambda}^{(0)}$ which is isomorphic to a self-dual $\mathcal{H}$-module $L^{\mathcal{H}}\left(\frac{c_{L}-2}{24}+\lambda, c_{L, I}\right)$.

\section{REFERENCES}

[1] E. Arbarello, C. De Concini, V. Kac, C. Procesi, Moduli spaces of curves and representation theory, Commun. Math. Phys. 117 (1988), 1-36.

[2] D. Adamović, G. Radobolja, Free field realization of the twisted Heisenberg-Virasoro algebra at level zero and its applications, Journal of Pure and Applied Algebra 219 (10) 2015, pp. 4322-4342

[3] D. Adamović, G. Radobolja, On free field realization of $W(2,2)$-modules, SIGMA 12 (2016), 113, 13 pages, arXiv:1605.08608

[4] D. Adamović, A. Milas, Lattice construction of logarithmic modules for certain vertex algebras, Selecta Mathematica, New Series 15 (2009) 535-561

[5] D. Adamović, A. Milas, On W-Algebras Associated to (2, p) Minimal Models and Their Representations, International Mathematics Research Notices 2010 (2010) 20 : 3896-3934

[6] D. Adamović, A. Milas, Vertex operator superalgebras and LCFT, Journal of Physics A: Mathematical and Theoretical. 46 (2013) , 49; 494005, Special Issue on Logarithmic conformal field theory

[7] D. Adamović R. Lu, K. Zhao, Whittaker modules for the affine Lie algebra $A_{1}^{(1)}$, Advances in Mathematics 289 (2016) 438-479, arXiv:1409.5354 
[8] B. Bakalov, Twisted logarithmic modules of vertex algebras, Commun. Math. Phys. 345 (2016), no. 1, 355-383.

[9] A. Bagchi, R. Gopakumar, Galilean conformal algebras and AdS/CFT, JHEP 07 (2009) 037, arXiv:0902.1385

[10] A. Bagchi, R. Gopakumar, I. Mandal, A. Miwa, GCA in 2D, JHEP 08 (2010) 004, arXiv:0912.1090

[11] N. Banerjee, D.P. Jatkar, S. Mukhi, T. Neogi, Free-field realisations of the BM $S_{3}$ algebra and its extensions, JHEP 06 (2016) 024, arXiv:1512.06240

[12] Y. Billig, Representations of the twisted Heisenberg-Virasoro algebra at level zero, Canadian Math. Bulletin, 46 (2003), 529-537, arXiv:0201314v1

[13] S. Berman, C. Dong and S. Tan, Representations of a class of lattice type vertex algebras, J. of Pure and Applied Algebra 176 (2002) 27-47

[14] H. Guo and Q. Wang, Twisted Heisenberg-Virasoro vertex operator algebra, arXiv:1612.06991.

[15] T. Creutzig, T. Gannon, Logarithmic conformal field theory, log-modular tensor categories and modular forms, arXiv:1605.04630

[16] E. Frenkel, Lectures on Wakimoto modules, opers and the center at the critical level, Adv. Math 195 (2005) 297-404.

[17] Y.-Z. Huang, Generalized twisted modules associated to general automorphisms of a vertex operator algebra, Commun. Math. Phys. 298 (2010), 265-292.

[18] Y.-Z. Huang, J. Lepowsky, L. Zhang, Logarithmic tensor category theory for generalized modules for a conformal vertex algebra, Parts I-VIII, arXiv:1012.4193, 1012.4196, 1012.4197, 1012.4198, 1012.4199, 1012.4202, 1110.1929, 1110.1931; Part I published in Conformal Field Theories and Tensor Categories, Springer, Berlin-Heidelberg, 2014. 169-248.

[19] Q. Jiang, C. Jiang, Representations of the twisted Heisenberg-Virasoro algebra and the full toroidal Lie algebras, Algebra Colloq., 2007, 14: 117-134.

[20] J. Lepowsky and H. Li, Introduction to Vertex Operator Algebras and Their Representations, Birkhäuser, Boston, 2003.

[21] F. Malikov, V. Schechtman, A. Vaintrob, Chiral de Rham complex, Comm. Math. Phys. 204 (1999) 439-473

[22] A. Milas, Weak modules and logarithmic intertwining operators for vertex operator algebras, in Recent Developments in Infinite-Dimensional Lie Algebras and Conformal Field Theory, ed. S. Berman, P. Fendley, Y.-Z. Huang, K. Misra, and B. Parshall, Contemp. Math., Vol. 297, American Mathematical Society, Providence, RI, 2002, 201-225.

[23] M. Miyamoto, Modular invariance of vertex operator algebra satisfying $C_{2}$-cofiniteness, Duke Math. J. 122 (2004) 51-91.

[24] G. Radobolja, Subsingular vectors in Verma modules, and tensor product of weight modules over the twisted Heisenberg-Virasoro algebra and W(2,2) algebra, Journal of Mathematical Physics 54071701 (2013)

[25] J. Rasmussen and C. Raymond, Galilean contractions of W-algebras, Nuclear Physics B, 922 435-479, arXiv:1701.04437

[26] A. Tsuchiya and Y. Kanie, Fock space representations of the Virasoro algebra - Intertwining operators Publ. Res. Inst. Math. Sci, 22:259- 327, 1986.

Current address: Department of Mathematics, Faculty of Science, University of Zagreb, Bijenička 30, 10 000 Zagreb, Croatia

E-mail address: adamovic@math.hr

Current address: Faculty of Science, University of Split, Ruđera Boškovića 33, 21000 Split, Croatia

E-mail address: gordan@pmfst.hr 